\title{
Comparison of PECARN, CATCH, and CHALICE clinical decision rules for pediatric head injury in the emergency department
}

\author{
Mark McGraw, BSc, MSc, MD*; Todd Way, BSc, MD ${ }^{\dagger}$
}

Clinical question: What is the diagnostic accuracy of the PECARN, CATCH, and CHALICE clinical decision rules for pediatric head injury, and are the clinical decision rules valid when applied to a novel data set?

Article chosen: Babl FE, Borland ML, Phillips $\mathrm{N}$, et al. Accuracy of PECARN, CATCH, and CHALICE head injury decision rules in children: a prospective cohort study. Lancet (London, England) 2017;389(10087):2393-402.

Objectives: The primary objective of the study was to determine the diagnostic accuracy and provide external validation for the PECARN, CATCH, and CHALICE clinical decision rules in a clinically homogeneous cohort of children. The secondary objective of this study was to perform a direct comparison of the three decision rules by assessing for the presence of traumatic brain injury (TBI) on computed tomography (CT) or the requirement for neurointervention.

Keywords: CATCH, CHALICE, clinical decision rules, emergency department, head injury, PECARN, pediatric head injury, pediatrics

\section{BACKGROUND}

Head trauma is one of the most common complaints in children presenting to emergency departments (EDs), and it is estimated that over 630,000 children present to EDs annually in the United States. ${ }^{1}$ Although most children presenting with a complaint of head trauma will not have a TBI, prompt neuroimaging by CT remains the gold standard in ruling out intracranial pathology. However, exposure to ionizing radiation during CT scans has been shown to increase rates of brain tumors and leukemias in children with an excess relative risk of 0.016 and 0.033 per mGy, respectively. ${ }^{2-4}$ While the absolute risk is low, overall rates of CT use in pediatric head injury are estimated to be $15 \%$ in Canada and $33 \%$ in the United States, creating significant population level risks. ${ }^{5-7}$ To prevent unnecessary exposure, standardize care, and reduce unnecessary healthcare expenditure, clinical decision rules are used to determine which patients require imaging. Prior to this study, the diagnostic accuracy of PECARN, CATCH, and CHALICE had not been directly compared or externally validated.

\section{POPULATION STUDIED}

This study enrolled all children under 18 years of age presenting to one of 10 pediatric EDs in Australia and New Zealand with head injuries of any severity. Exclusion criteria for the study were patients who presented with trivial facial injuries only, were referred from triage directly to an external provider, received neuroimaging prior to being transferred to a study site, or did not wait to be seen.

\section{STUDY DESIGN}

The study was a prospective multicentre observational study conducted in Australia and New Zealand from April 11, 2011, to November 30, 2014. Data were collected on all children that met inclusion criteria. For each rule, the authors recorded the inclusion and exclusion criteria, predictor variables, and outcome measures for each study participant. Follow-up telephone interviews were conducted with all patients in the study who did not receive neuroimaging at the time of presentation, and data were

From the * Memorial University of Newfoundland School of Medicine, St. John's, NL; and tDepartment of Emergency Medicine, Saint John Regional Hospital, St. John, NB.

Correspondence to: Dr. Mark McGraw, Medical Education 3DS, PO Box 2100, Saint John, NB E2L 4L2; Email: mark.mcgraw@dal.ca 
Comparison of PECARN, CATCH, and CHALICE clinical decision rules for pediatric head injury in the ED

CHALICE Clinical Decision Rule
History
1. Witnessed LOC $>5$ minutes
2. History of amnesia $>5$ minutes
3. Abnormal drowsiness
4. Over 3 discrete vomits
5. Physician suspicion of nonaccidental
injury
6. First ever seizure after injury
Examination
7. GCS $<14$ or $<15$ if under 1 year
8. Suspicion of penetrating or depressed
skull injury or tense fontanelle.
9. Signs of basil skull fracture.
10 . Positive focal neurological finding.
11 . Presence of bruise, swelling, or
laceration $>5 \mathrm{~cm}$ if $<1$ year old.
Mechanism
12 . Dangerous mechanism (MVA $>$
$40 \mathrm{mph}$, fall $>3$ meters, high speed
projectile injury.

CATCH Clinical Decision Rule

CT head is required only for children with minor head injury (injury within the past 24 hours associated with witnessed LOC, definite amnesia, witnessed disorientation, persistent vomiting, or persistent irritability) plus any one of the following

High Risk:

1. GCS $<15$ two hours post injury. 2. Suspected open or depressed skull fracture.

3. History of worsening headache.

4. Irritability on examination.

Medium Risk:

5. Any sign of basal skull fracture.

6. Large, boggy hematoma of the scalp.

7. Dangerous mechanism (MVA, fall >

$3 \mathrm{ft} / 0.9 \mathrm{~m}$ or from 5 stairs, fall from

bicycle with no helmet)
PECARN : Children younger than 2 years

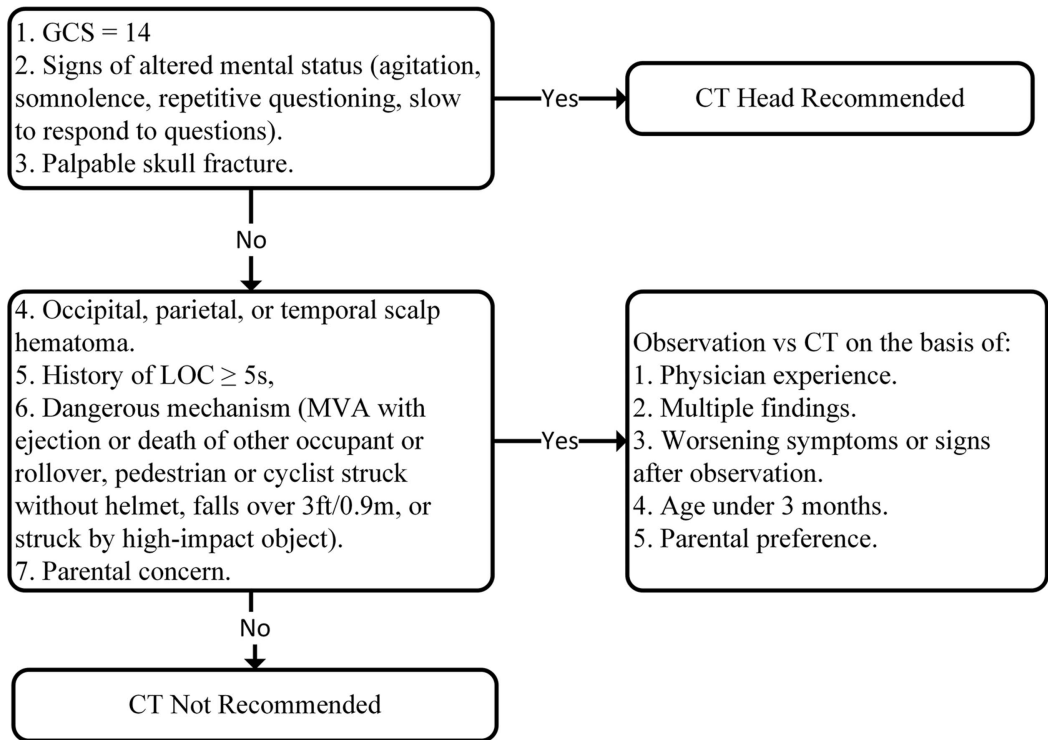

PECARN : Children 2 years and older

1. $\mathrm{GCS}=14$

2. Signs of altered mental status (agitation, somnolence, repetitive questioning, slow

to respond to questions).

CT Head Recommended

3. Signs of basilar skull fracture.

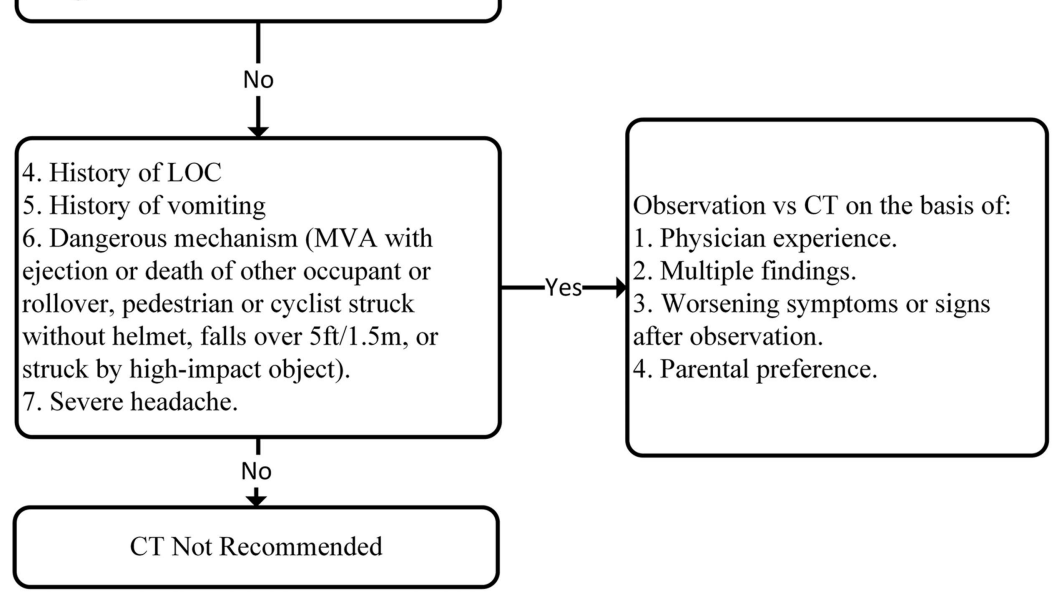

Figure 1. PECARN, CATCH, and CHALICE Clinical Decision rules for pediatric head injury.

recorded for any patients with subsequent presentations resulting in neuroimaging.

\section{OUTCOME MEASURES}

The primary outcome was the diagnostic accuracy of each clinical decision rule, according to its own rule-specific outcome: clinically significant TBI (cTBI) for PECARN, need for neurological intervention in $\mathrm{CATCH}$, and clinically significant intracranial injury in CHALICE. The secondary outcome of the study was a comparison of the ability of each rule to predict a standardized outcome of cTBI in a homogeneous cohort of children.

\section{RESULTS}

During the study period, 29,433 patients under age 18 presented with head injuries to the participating 
EDs, and 20,137 were eligible for analysis. The mean age was 5.7 years, $63.7 \%$ were male, and $95.4 \%$ of the patients presented with a Glasgow Coma Scale of 15 . The most common mechanism of injury was a fall $(70.1 \%)$, and the most common symptoms were headache $(20.5 \%)$, vomiting $(17.1 \%)$, or witnessed disorientation (14.6\%); 2,106 (10\%) patients received a CT scan, 4,544 (23\%) were admitted to a hospital (inpatient ward, short-stay ward, or intensive care unit), $83(<1 \%)$ received neurosurgery, and $15 \quad(<1 \%)$ died.

PECARN criteria were applicable for 4,011 children under age 2 years and 11,152 patients over age 2 years. Criteria were positive for 1,872 children under age 2 years, 42 of whom had cTBI, and there were no missed cases of cTBI in this group. In the PECARN group over age 2 years, 6,084 children met the criteria, and 117 had cTBI. One study participant with cTBI was missed in the PECARN group over 2 years of age. The missed patient had signs of a basilar skull fracture and would have been positive in the CHALICE criteria but also missed in CATCH.

High-risk criteria for CATCH were applicable in 4,957 patients, and 799 of the patients had a positive decision rule. Neurological intervention was required for 20 patients who were positive for the rule and 1 patient negative for the rule. Combined highand medium-risk criteria from CATCH were also applicable for 4,957 patients, and 2,225 were positive for the combined criteria. Brain injury on CT was found in 125 patients who were screened positive for the rule and 16 who were screened negative and were missed.

CHALICE criteria were applicable for 20,029 patients in the study, with 4,673 patients screening positive for the criteria. Clinically significant intracranial injury occurred in 370 of the patients who were positive for the rule and 31 patients were negative for the rule, two of whom required neurosurgery.

The diagnostic accuracy of the rules is given in Table 1. PECARN had the highest sensitivity of the three rules at $100.0 \%$ and $99.0 \%$ for children ages $<2$ years and $\geq 2$ years, respectively.

Results of the secondary outcome for the study are given in Table 2. PECARN missed the fewest patients and had the highest sensitivity overall; however, confidence intervals on all three rules overlapped, indicating that none of the rules were superior in this cohort.

\section{Validity of results}

This study was a large prospective multicentre observational study with a robust design, clear selection criteria, and appropriate power to provide external validation of the rules. The study was not designed to compare the rules statistically, because the three rules have fundamentally different end points: PECARN was designed to identify children at very low risk of cTBI who do not require a CT, whereas CHALICE and CATCH were designed to identify children at high risk who would benefit from the use of a CT scan.

Objections to the direct comparison of the rules due to the difference in their outcomes were published in a subsequent issue of The Lancet from the authors of CATCH and PECARN. ${ }^{8,9}$ The authors of this study acknowledge this discrepancy but suggest that the results of the study are clinically relevant as they address the pragmatic question of what clinical decision rule a physician should use to determine whether a pediatric patient presenting with a head injury requires a CT. ${ }^{10}$ The authors used a reasonable approach to objectively compare the rules by ensuring that data were collected to appropriately evaluate each rule and that follow-up was sufficient. Despite the difference in their intended use, the direct comparison of the diagnostic accuracies amongst the rules on a novel data set compiled using each rules inclusion and exclusion criteria, predictor variables, and outcomes still provides clinicians with clinically relevant information.

\section{COMMENTARY}

All three rules had excellent sensitivities and performed well in assessing the secondary outcome of cTBI, suggesting that all were appropriate for use in assessing mild head injury in the ED. However, application of the rules to the study participants would have resulted in large differences in CT rates for patients. The baseline rate for CT in this study was $8.3 \%$. Application of the CHALICE or CATCH rules would have resulted in an adjusted CT rate of $22.0 \%$ or $30.2 \%$, an increase of $165.1 \%$ to $263.9 \%$, respectively. Unfortunately, determining the rates of CT use by a retrospective application of PECARN criteria is not possible. However, preliminary studies assessing the impact of implementing PECARN criteria on CT rates have demonstrated reduced rates, in centres with high baseline CT rates, or stable rates in 
Comparison of PECARN, CATCH, and CHALICE clinical decision rules for pediatric head injury in the ED

\begin{tabular}{|c|c|c|c|c|c|}
\hline & \multicolumn{2}{|c|}{ PECARN } & \multicolumn{2}{|c|}{$\mathrm{CATCH}$} & \multirow{2}{*}{$\begin{array}{c}\text { CHALICE } \\
\text { All patients }\end{array}$} \\
\hline & $<2$ years & $\geq 2$ years & All patients & All patients & \\
\hline & $n=4,011$ & $n=11,152$ & $n=4,957$ & $n=4,957$ & $n=20,029$ \\
\hline $\begin{array}{l}\text { Outcome } \\
\text { assessed } \\
\text { Positive } \\
\text { decision } \\
\text { rule }\end{array}$ & $\begin{array}{l}\text { Clinically important } \\
\text { traumatic brain injury }\end{array}$ & $\begin{array}{l}\text { Clinically important } \\
\text { traumatic brain injury }\end{array}$ & $\begin{array}{l}\text { Neurological } \\
\text { intervention }\end{array}$ & Brain injury on $\mathrm{CT}$ & $\begin{array}{l}\text { Clinically significant } \\
\text { intracranial injury. }\end{array}$ \\
\hline $\begin{array}{l}\text { With } \\
\text { outcome (n) }\end{array}$ & 38 & 97 & 20 & 125 & 370 \\
\hline $\begin{array}{l}\text { Without } \\
\text { outcome (n) } \\
\text { Negative } \\
\text { decision } \\
\text { rule }\end{array}$ & 1,834 & 5,987 & 779 & 2,100 & 4,303 \\
\hline $\begin{array}{l}\text { With } \\
\text { outcome (n) }\end{array}$ & 0 & 1 & 1 & 16 & 31 \\
\hline $\begin{array}{l}\text { Without } \\
\text { outcome (n) }\end{array}$ & 2,139 & 5,067 & 4,157 & 2,716 & 15,352 \\
\hline $\begin{array}{l}\text { Sensitivity } \\
(95 \% \mathrm{Cl})\end{array}$ & $100.0 \%(90.7-100.0)$ & $99.0 \%(94.4-100.0)$ & $95.2 \%(76.2-99.9)$ & $88.7 \%(82.2-93.4)$ & $92.3 \%(89.2-94.7)$ \\
\hline $\begin{array}{l}\text { Specificity } \\
(95 \% \text { Cl) }\end{array}$ & $53.8 \%(52.3-55.4)$ & $45.8 \%(44.9-46.8)$ & $84.2 \%(83.2-85.2)$ & $56.4 \%(55.0-57.8)$ & $78.1 \%(77.5-78.7)$ \\
\hline PPV (95\% CI) & $2.0 \%(1.4-2.8)$ & $1.6 \%(1.3-1.9)$ & $2.5 \%(1.5-3.8)$ & $5.6 \%(4.7-6.7)$ & $7.9 \%(7.2-8.7)$ \\
\hline NPV (95\% Cl) & $100.0 \%(99.8-100.0)$ & $100.0 \%(99.9-100.0)$ & $100.0 \%(99.9-100.0)$ & $99.4 \%(99.1-99.7)$ & $99.8 \%(99.7-99.9)$ \\
\hline
\end{tabular}

\begin{tabular}{|c|c|c|c|c|}
\hline & PECARN & & CATCH & CHALICE \\
\hline & $<2$ years & $\geq 2$ years & All patients & All patients \\
\hline & $n=5,046$ & $n=13,867$ & $n=18,913$ & $n=18,913$ \\
\hline \multicolumn{5}{|c|}{ Outcome assessed: Clinically important TBI } \\
\hline Sensitivity $(95 \% \mathrm{Cl})$ & $100.0 \%(91.6-100.0)$ & $99.2 \%(95.4-100.0)$ & $91.9 \%(86.5-95.6)$ & $92.5 \%(87.3-96.1)$ \\
\hline Specificity $(95 \%$ Cl) & $59.1 \%(57.7-60.5)$ & $52.0 \%(51.1-52.8)$ & $70.4 \%(69.7-71.0)$ & $78.6 \%(78.0-79.2)$ \\
\hline Missed & 0 & 1 & 13 & 12 \\
\hline \multicolumn{5}{|c|}{ Outcome assessed: Traumatic brain injury on CT } \\
\hline Sensitivity $(95 \% \mathrm{Cl})$ & $100.0 \%(94.9-100.0)$ & $99.4 \%(97.0-100.0)$ & $87.6 \%(82.9-91.5)$ & $90.4 \%(86.1-93.8)$ \\
\hline Specificity $(95 \% \mathrm{Cl})$ & $59.4 \%(58.0-60.8)$ & $52.2 \%(51.4-53.0)$ & $70.6 \%(69.9-71.3)$ & $78.9 \%(78.3-79.5)$ \\
\hline Missed & 0 & 1 & 31 & 24 \\
\hline \multicolumn{5}{|c|}{ Outcome assessed: Neurosurgery } \\
\hline Sensitivity $(95 \% \mathrm{Cl})$ & $100.0 \%(54.1-100.0)$ & $100.0 \%(81.5-100.0)$ & $95.8 \%(78.9-99.9)$ & $91.7 \%(73.0-99.0)$ \\
\hline Specificity (95\% Cl) & $58.7 \%(57.3-60.0)$ & $51.6 \%(50.7-52.4)$ & $69.9 \%(69.2-70.6)$ & $78.1 \%(77.5-78.6)$ \\
\hline Missed & 0 & 0 & 1 & 2 \\
\hline
\end{tabular}


centres with baseline CT rates below $10 \% .{ }^{11-14}$ It is suggested that the reduced rates are linked to the rules inclusion of an option for physician observation in the $\mathrm{ED}$, which has been shown independently to reduce CT rates without worsening outcomes for patients. ${ }^{15}$

\section{CONCLUSION}

The results of this study provide external validation of the PECARN, CATCH, and CHALICE clinical decision rules. Application of the PECARN clinical decision rule resulted in the fewest missed patients with cTBI. The analysis of the comparison cohort suggests that the PECARN rule had a higher sensitivity for identifying cTBI; however, the $95 \%$ confidence intervals of all three rules overlapped, and none could be deemed superior.

Competing interests: None declared.

\section{REFERENCES}

1. Thurman DJ. The epidemiology of traumatic brain injury in children and youths: a review of research since 1990. 7 Child Neurol 2016;31(1):20-7.

2. Brenner DJ, Hall EJ. Computed tomography - an increasing source of radiation exposure. $N$ Engl 7 Med 2007;357 (22):2277-84.

3. Brenner DJ. Estimating cancer risks from pediatric CT: going from the qualitative to the quantitative. Pediatr Radiol 2002;32(4):228-31.

4. de Gonzalez AB, Salotti JA, McHugh K, et al. Relationship between paediatric CT scans and subsequent risk of leukaemia and brain tumours: assessment of the impact of underlying conditions. Br 7 Cancer 2016;114:388.
5. Pearce MS, Salotti JA, Little MP, et al. Radiation exposure from CT scans in childhood and subsequent risk of leukaemia and brain tumours: a retrospective cohort study. Lancet 2012;380(9840):499-505.

6. Klassen TP, Reed MH, Stiell IG, et al. Variation in utilization of computed tomography scanning for the investigation of minor head trauma in children: a Canadian experience. Acad Emerg Med 2008;7(7):739-44.

7. Stanley RM, Hoyle JD, Dayan PS, et al. Emergency department practice variation in computed tomography use for children with minor blunt head trauma. 7 Pediatr 2014;165(6):1201-6.e2.

8. Osmond MH. Head injury decision rules in children. Lancet 2017;390(10101):1487.

9. Kuppermann N, Holmes JF, Dayan PS. Head injury decision rules in children. Lancet 2017;390(10101):1487-8.

10. Babl FE, Oakley E, Borland ML, et al. Head injury decision rules in children - authors' reply. Lancet 2017;390 (10101):1488.

11. Bressan S, Romanato S, Mion T, et al. Implementation of adapted PECARN decision rule for children with minor head injury in the pediatric emergency department. Acad Emerg Med 2012;19(7):801-7.

12. Lorton F, Poullaouec C, Legallais E, et al. Validation of the PECARN clinical decision rule for children with minor head trauma: a French multicenter prospective study. Scand 7 Trauma Resusc Emerg Med 2016;24(1):98.

13. Ide K, Uematsu S, Tetsuhara K, et al. External validation of the PECARN head trauma prediction rules in Japan. Acad Emerg Med 2017;24(3):308-14.

14. Nigrovic LE, Stack AM, Mannix RC, et al. Quality improvement effort to reduce cranial CTs for children with minor blunt head trauma. Pediatrics 2015;136: e227.

15. Schonfeld D, Fitz BM, Nigrovic LE. Effect of the duration of emergency department observation on computed tomography use in children with minor blunt head trauma. Ann Emerg Med 2013;62(6):597-603. 